\title{
LONG-TERM EVOLUTION OF (SHORT) GAMMA-RAY BURST CENTRAL ENGINES
}

\author{
William H. Lee ${ }^{1}$ \\ RESUMEN
}

Los destellos de rayos gama cosmólogicos posiblemente provienen de discos de acreción alrededor de agujeros negros. Estos pueden ser producto de una fusión binaria de objetos compactos. Resulta importante estudiar la evolución dinámica de estos discos si se quiere relacionar las escalas temporales características con la duración y variabilidad observada en destellos. Mostramos resultados de tales cálculos, relevantes para destellos cortos.

\section{ABSTRACT}

Cosmological gamma ray bursts (GRBs) possibly originate from accretion disks around stellar mass black holes. These could be formed after the merger of a double neutron star or black hole-neutron star binary. The dynamical cvolution of the disk is important if one wishes to relate characteristic timescales with the observed duration and variability. We show here the results of such a set of calculations, relevant for short GRBs.

\section{Key Words: ACCRETION, ACCRETION DISKS - GAMMA RAYS: BURSTS - HYDRODYNAMICS - STARS: NEUTRON}

\section{INTRODUCTION}

The extreme form of a compact binary, the subject matter of this meeting, is one that contains two compact objects, such as neutron stars (NS) or black holes $(\mathrm{BH})$, and where the separation is comparable to the stellar radii. Systems with two black holes will lack an electromagnetic signal, and reveal their nature only through the emission of gravitational waves (Thorne 1995). If at least one of the components is not a BH, a combined burst of gravitational and electromagnetic energy can be expected. This is the fate of systems such as the recently discovered PSR J0737-3039 and the famous Hulse-Taylor binary pulsar (Burgay et al. 2003; Hulse \& Taylor 1975).

Simply on energetic grounds, double NS systems or BH-NS binaries have been considered as possible progenitors of cosmological gamma ray bursts, particularly for the short variety, releasing $\sim 10^{52}$ erg in a few seconds (Lattimer \& Schramm 1976; Paczyński 1986). Currently, the most favored model for long bursts involves the core collapse of a massive star (Woosley 1993). How this energy is released, and converted to the prompt gamma rays we observe as a burst remains to be clarified. However, it is obvious that elucidating the details requires modeling the putative central engine for a time that is comparable with the duration of the burst itself, i.e. a few tenths of a second for short GRBs, and several tens of seconds for long ones.

The binary coalescence process is instrinsically

\footnotetext{
${ }^{1}$ Instituto de Astronomía, UNAM, México.
}

three-dimensional, and requires an extensive amount of computational resources. Recent 3D calculations of the merger itself, or of the subsequent disk evolution, do not cover more than 20-60 ms (Kluźniak \& Lee 1998; Lee 2001; Rosswog, Speith \& Wynn 2004; Setiawan, Ruffert \& Janka 2004). They can thus determine how an accretion disk might be formed, but cannot address its long-term dynamics. They can, however, be used as initial conditions for a diferent set of calculations, which cover a longer period.

\section{EVOLUTION OF THE CENTRAL ENGINE}

The simplest way to extend the period covered by the simulations is to sacrifice one dimension, mapping the $3 \mathrm{D}$ output from binary merger calculations to 2D by assuming azimuthal symmetry (Lee \& Ramirez-Ruiz 2002). This approximation is quite good, as can be seen by inspecting the mass distribution at late times in such simulations. At the densities and temperatures encountered in such a configuration, photons are completely trapped in the fluid, and the main cooling agent is neutrino emission. The results can be made physically meaningful by: (1) using a detailed equation of state; (2) modeling angular momentum transport with an $\alpha$-type prescription for viscosity and (3) including the proper neutrino emission rates as a cooling term. Further, the densities are high enough that neutrino scattering off free nucleons is significant, and a "neutrino-surface" is present within the disk, at about $\rho \sim 10^{11} \mathrm{~g} \mathrm{~cm}^{-3}$. This can be accounted for in a simple manner by suppressing the cooling rate by $\exp \left[-\tau_{\nu}\right]$, where $\tau_{\nu}$ 


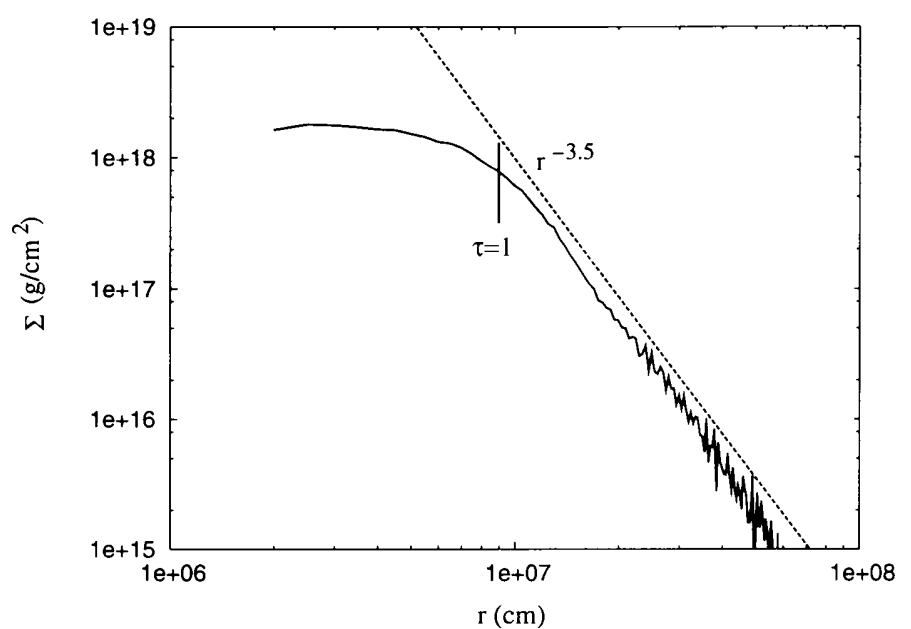

Fig. 1. Surface density profile $0.2 \mathrm{~s}$ after the start of the calculation for a run with $\alpha=0.01$. The position where $\tau_{\nu}=1$ is indicated with a vertical mark, at $r \sim$ $9 \times 10^{6} \mathrm{~cm}$.

is the optical depth for neutrinos. Initially the $\mathrm{BH}$ harbors $\sim 4 \mathrm{M}_{\text {ollot }}$, and the disk holds $\sim 0.3 \mathrm{M}_{\odot}$. Our calculations typically cover $\sim 0.5 \mathrm{~s}$.

We have carried out a set of simulations under these assumptions (Lee, Ramirez-Ruiz \& Page 2004), using a Smooth Particle Hydrodynamics code (Monaghan 1992) (in three dimensions for the merger process, and two for the disk evolution), with various magnitudes for the viscosity $(0.001 \leq \alpha \leq 0.1)$. We find important characteristic timescales in the resulting time evolution of the neutrino luminosities and maximum densities in the disks, as well as in their spatial structure.

For low enough viscosities $\left(\alpha \leq 10^{-2}\right)$, the disk is not depleted of its mass quickly, and radiates away all of its internal energy on a cooling timescale $t_{\text {cool }} \sim E_{\text {int }} / L_{\nu} \sim 0.1 \mathrm{~s}$. The presence of an optically thick region (to neutrinos) in its center is crucial, as it allows the energy release to be spread over an extended period of time $\left(L_{\nu} \sim 10^{53} \mathrm{erg}\right)$. The spatial structure of the disk is affected as well by this opaque region. The surface density profile is roughly $\Sigma\left[\tau_{n u}>1\right] \propto$ cst, and $\Sigma\left[\tau_{n u}<1\right] \propto$ $r^{-3.5}$. The break occurs at $r \sim 10^{7} \mathrm{~cm}$, where $\rho \sim 10^{11} \mathrm{~g} \mathrm{~cm}^{-3}$. This dense region could in principle anchor strong magnetic fields (the equipartition value is $\sim 10^{15}-10^{16} \mathrm{G}$ ), which would launch strong outflows, possibly resulting in a GRB. The timescale for a change in the global structure of the disk's magnetic field can be estimated as $t_{j e t} \sim t_{d y n} 2^{R / H}$ (Livio, Pringle \& King 2003), where $t_{d y n}$ is the dy- namical timescale in the inner regions $(\sim 2-5 \mathrm{~ms})$. and $H$ is the pressure scale height. In our calculations, $H / R \sim 0.3$, which gives $t_{\text {jet }} \sim 10-100 \mathrm{~ms}$ (Lee, Ramirez-Ruiz \& Page 2004). This is of the order of the variability timescale seen in short GRBs (Nakar \& Piran 2002).

A number of previous studies have addressed the structure of accretion disks under similar conditions. assuming a steady state (Popham, Woosley \& Fryer 1999; Narayan, Piran \& Kumar 2001: Kohri \& Mineshige 2002; Di Matteo, Perna \& Narayan 2002). This, while clearly very useful, obviously requires an external source of matter, which is not entirely justified in the case of a disk originating from a binary merger. Our results indicate that the dynamics of such disks may be fundamentally related to the variability and duration of short gamma ray bursts.

It is a pleasure to acknowledge collaboration in this work with W. Kluźniak, D. Page and E. Ramírez-Ruiz.

\section{REFERENCES}

Burgay, M. et al. 2003, Nature, 426, 531

Di Matteo, T., Perna, R. \& Narayan, R. 2002, ApJ, 579, 706

Hulse, R. A. \& Taylor, J. H. 1975, ApJ, 195, L51

Kluźniak, W. \& Lee, W. H. 1998, ApJ, 494, L53

Kohri, K. \& Mineshige, S. 2002, ApJ, 577, 311

Lattimer, J. M. \& Schramm, D. N. 1976, ApJ, 210, j49

Lee, W. H. 2001, MNRAS, 328, 583

Lee, W. H. \& Ramirez-Ruiz, E. 2002, ApJ, 577, 893

Lee, W. H., Ramirez-Ruiz, E. \& Page, D. 2004, ApJ Letters submitted

Livio, M., Pringle, J. E., \& King, A. R. 2003, ApJ, 593. 184

Monaghan, J. J. 1992, ARAA, 30, 543

Nakar, E. \& Piran, T. 2002, MNRAS, 330, 920

Narayan, R., Piran, T. \& Kumar, P. 2001, ApJ. 557, 949

Paczyński, B. 1986, ApJ, 308, L43

Popham, R., Woosley, S. E. \& Fryer, C. 1999. ApJ, 518. 356

Rosswog, S., Speith, R. \& Wynn, G. A. 2004. MNRAS submitted (astro-ph/0403500

Setiawan, S., Ruffert, M. \& Janka, H.-Th. 2004, MNRAS submitted (astro-ph/0402481)

Thorne K. S., 1995, in Kolb E. W., Peccei R., eds., Proceedings of the Snowmass 95 Summer Study on Particle and Nuclear Astrophysics and Cosmology. World Scientific, Singapore

Woosley, S. E. 1993, ApJ, 405, 273

William H. Lee: Instituto de Astronomía, UNAM, Apdo. Postal 70-264, México, D. F. México (wlee@astroscu.unam.mx). 